\title{
ANOMALOUS BEHAVIOR OF LIQUIDS IN ISOLATED CONTACT WITH SOLIDS ${ }^{\dagger}$
}

\author{
WILLIAM J. O'BRIEN \\ University of Michigan*, School of Dentistry, Ann Arbor, Michigan 48104,U.S.A. \\ and \\ Marquette University, Milwaukee, Wisconsin, U.S.A.
}

Received 2 April 1970; revised manuscript received 20 October 1970

Anomalous liquid behavior is viewed as a result of an increase in surface tension in isolated capillaries. Using a derived prime equation for this change several phenomena are treated including the strength of liquid bridges between solids, the lowering of vapor pressure, increased solubility, and adsorption-desorption hysteresis. Using experimental data available on the strength of liquid bridges and vapor pressure decreases, indicates the surface tension of water in micron sized capillaries has a surface tension of over 700 dyne $/ \mathrm{cm}$.

\section{Isolated capillary theory}

It has become apparent in recent years that classical capillary theory is not able to explain several established experimental observations. Since 1928, Shereshefsky ${ }^{1,2}$ ) with several co-workers has demonstrated large vapor pressure reductions with a variety of liquids in capillaries a few microns in radii. $\Lambda \mathrm{lso}$, the phenomena of adsorption desorption hysteresis has not been explained satisfactorily. Several theories have been proposed, but none verified.

Recently, the Fisher equation for the strength of liquid bridges has been questioned $^{3}$ ). This equation is based on the contributions of both a surface tension force and a reduced pressure component. Whereas other capillary phenomena employ these contributions separately. However, the Fisher equation has been verified experimentally. The most recent breakdown in classical capillary theory had been in the anomalous water controversy ${ }^{4}$ ). Anomalous water is prepared by the condensation of unsaturated water vapor in micron size glass and quartz tubes and therefore is a capillary effect.

† This research was supported in part by USPHS Research Grant DE 01057 from the National Institute of Dental Research, National Institutes of Health, Bethesda, Maryland to Marquette University.

* Present address. 
All of these contested phenomena involve liquids in small capillaries that are isolated from a reservoir. The response of such a system to liquid-solid wetting could not be the same as liquid drops or connected capillaries. In a recent paper by $\mathrm{O}^{\prime}$ Brien $^{5}$ ), the surface tension of the liquid in isolated capillaries is postulated to change as follows,

$$
\mathrm{d} \gamma / \mathrm{d} A_{\mathrm{SL}}=\left(F_{\mathrm{SV}}-F_{\mathrm{SL}}\right) / A_{\mathrm{LV}}
$$

where $\left(F_{\mathrm{SV}}-F_{\mathrm{SL}}\right)$ represents the free energy change on wetting and $A_{\mathrm{SL}}$ and $A_{\text {Lv }}$ are the solid-liquid and liquid-vapor interfacial areas. This equation may be integrated for the case of a closed ended cylinder of radius $r$ filled to a height $h$ :

$$
\gamma^{*}-\gamma=h \gamma \cos \theta / r
$$

\section{Experimental evidence}

\subsection{StRENGTH OF LIQUID BRIDGES}

When a thin film of liquid is placed between two particles it exhibits high tensile strength. This is one example of the anomalous behavior of liquids in isolated contact with solids. The increase of strength of the liquid may be treated as a result of an increased surface tension. For two spheres of radius $R$ separated by a liquid bridge which subtends an angle $\psi$ from the sphere's center, an equation for the force of adhesion, $f$, would be:

$$
f=2 \pi R \sin \psi\left[\gamma+\left(F_{\mathrm{SV}}-F_{\mathrm{SL}}\right) A_{\mathrm{SL}} / A_{\mathrm{LV}}\right] .
$$

Making simplifying assumption about $A_{\mathrm{SL}}$ and $A_{\mathrm{LV}}$ along with the use of equation (3) leads to the Fisher equation:

$$
f=2 \pi R \gamma \sin \psi+\gamma \cos \theta / r \cdot \pi R^{2} \sin ^{2} \psi,
$$

without introducing the concept of a $\Delta P$ or negative pressure. However, the term $\gamma \cos \theta / r$ may be expressed as $\Delta P$. A more detailed proof is given in ref. 5 .

Several experimental studies have verified the predictability of the Fisher equation. Cross and Picknett published one in which the force of adhesion between two spheres was measured as a function of the thickness of a water bridge $^{6}$ ). Their data may be used to calculate the apparent surface tension of the liquid film with the equation,

$$
f=2 \pi R \sin \psi \gamma^{*} .
$$

The results are shown in fig. 1.

The highest force of adhesion measured indicates a surface tension of several hundred dyne/cm. 
The classical capillary approach ascribes the increase in adhesion found with liquid bridges to a reduced pressure beneath the meniscus compared to the atmosphere ${ }^{7}$ ). If this were the case, the maximum adhesion possible would be $1 \times 10^{6}$ dyne $/ \mathrm{cm}^{2}(1 \mathrm{~atm})$ and would be altitude dependent. It is more reasonable to credit this increase in adhesion to a decrease in pressure within the liquid resulting in an increased inward attraction of the surface molecules. This is equivalent to a higher surface energy and surface tension in the case of liquids.

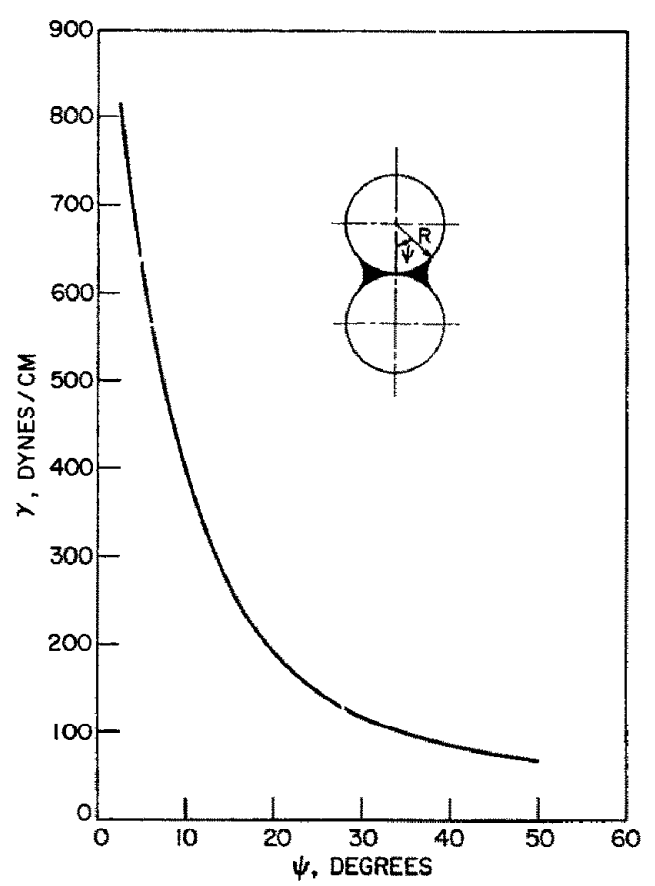

Fig. 1. Apparent surface tension as a function of angle to liquid bridge. Calculated from adhesion values of Cross and Picknett ${ }^{6}$ ).

\subsection{LARGE YAPOR PRESSURE REDUCTION}

The data of Shereshefsky ${ }^{8}$ ) for reduced vapor pressure in capillaries are in reasonable agreement with the Kelvin equation if calculated values of $\gamma^{*}$ are used as follows,

$$
R T \ln \left(P^{*} / P_{0}\right)=2 \gamma^{*} V / r
$$

for an isolated liquid. A problem does exist in calculating $\gamma^{*}$ since $A_{\text {SL, }}$ values are not available from Shereshefsky's experiments. However, he used tubes and gives the radius from which table 1 may be calculated for values of $h$ using eq. (2). An $h$ value of $10 \mu \mathrm{m}$ is sufficient to produce a vapor 
pressure lowering of 10 times. Using the value of $72 \mathrm{dyne} / \mathrm{cm}$, the calculated $\Delta P$ value is 0.017 . Shereshefsky reports values of $10-30$ for $\Delta P$. As in the case of liquid bridge strength, this effect is a strong one. Based on his data, the $\gamma^{*}$ of the water was at least $766 \mathrm{dyne} / \mathrm{cm}$.

TABLE 1

(Water at $18^{\circ} \mathrm{C}, P_{0}=15.477 \mathrm{~mm}$ )

\begin{tabular}{crcc}
\hline$h$ (microns) & $\gamma^{*}$ & $P^{*} / P_{0}$ & $\Delta P$ \\
\hline 0 & 72 & 0.9989 & 0.017 \\
1 & 130 & 0.9981 & 0.030 \\
2 & 198 & 0.9971 & 0.046 \\
5 & 411 & 0.9941 & 0.092 \\
10 & 766 & 0.9886 & 0.176 \\
\hline
\end{tabular}

\subsection{INCREASED SOLUBILITY}

An increase in surface tension of a liquid placed in an isolated capillary should increase its dissolving power since,

$$
\delta=4.1\left(\gamma / V^{\frac{1}{3}}\right)^{0.43},
$$

where $\delta$ is the solubility parameter of a nonpolar liquid, $\gamma$ is the surface tension and $V$ is the molar volume of the liquid ${ }^{9}$ ).

An equation giving the increase in solubility of a sparingly soluble salt in a liquid in an isolated capillary may be derived as follows. Consider the free energy difference in forming ion sized holes in a liquid of increased surface tension:

$$
\Delta F=(2 V / r)\left(\gamma^{*}-\gamma\right),
$$

where $V$ is the molar free volume and $r$ is the radius of the ion sized hole. The difference in activities in ions produced by an increase in surface tension of a liquid is then equated to this free energy difference to give the equation,

$$
R T \ln \left(a^{*} / a_{0}\right)=2 V\left(\gamma^{*}-\gamma\right) / r .
$$

If a salt dissolves to form $n$ positive ions and $m$ negative ions, the difference in solubilities is then,

$$
R T(n+m) \ln \left(S^{*} / S_{0}\right)=2 V\left(\gamma^{*}-\gamma\right) / r .
$$

This equation indicates an increase in solubility of a salt in a liquid whose surface tension has been increased. For silver chloride, assuming a $\Delta \gamma$ of $600 \mathrm{dyne} / \mathrm{cm}$ and an ionic radius of $2 \AA$,

$$
\begin{aligned}
\ln \left(S^{*} / S_{0}\right) & =(0.4343)(34)(600) /\left(8.3+10^{7}\right)(300)\left(2 \times 10^{-8}\right)=17.8, \\
S^{*} / S_{0} & =6.3 \times 10^{17}
\end{aligned}
$$


This large ratio indicates that the walls of a small isolated capillary would be subject to increased dissolution. A series of carefully controlled experiments has verified this phenomena. Rabideau and Florin ${ }^{10}$ ) collected condensate in $2-70 \mu \mathrm{m}$ diameter quartz capillaries from triple distilled water. An electron probe analysis of the concentrated residues indicated a high weight fraction of sodium, boron and oxygen. Neutron activation analysis and mass spectrometric measurements showed the presence of about $6 \%$ boron.

Davies, Rousseau, and Board ${ }^{11}$ ) used electron spectroscopic analysis to study 15 samples of water condensed in isolated capillaries and found large concentrations of sodium, potassium, sulfate, nitrate, carbonate, borates and silicates.

Rousseau and Porto ${ }^{12}$ ) analyzed condensates similarly prepared and found $20-60 \%$ sodium, $15 \%$ chlorine, $15 \%$ sulphate, $3 \%$ potassium and $1 \%$ calcium.

A possible mechanism for the dissolution of capillary walls by the condensing water is depicted in fig. 2. The water dipole chain is similar to that visualized for interface water or viseral water proposed by Drost-Hansen ${ }^{13}$ ).

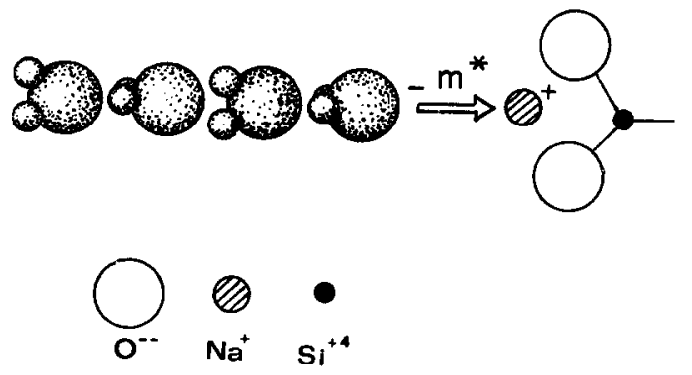

Fig. 2. Possible mechanism for solution of capillary walls by water polypolechain with dipole vector $m^{*}$.

\subsection{ADSORPTION-DESORPTION HYSTERESIS}

A difference in the adsorption and desorption cycles found with porous materials is a widespread phenomena and is illustrated in fig. 3 .

The isolated capillary theory may be applied to this phenomena as follows. As the pores illustrated in fig. 4, fill up with condensate, the solid wall material dissolves. The rate of solution is proportioned to $\Delta \gamma$ which depends on geometry as illustrated in fig. 5. Higher concentrations of dissolved solid would then form in the inverted cone and spherical pores.

After the adsorption cycle is completed, the solution exhibits a lower vapor pressure and subsequent desorption proceeds at a slower rate. Desorption becomes a concentrating process since solvent is evaporated. When 
the pores are half emptied, the concentration of dissolved solute would be greater than at a given level during adsorption. The change in vapor pressure with $n_{2}$ moles of solute would be,

$$
\Delta P / P_{0}=n_{2} / \sum n \text {. }
$$

Since $n_{2}$ is higher during desorption, $\Delta P$ will be greater.

This explanation is attractive since the dissolving of tube walls has been clearly demonstrated during the polywater controversy. Also true hysteresis would result since the adsorption process is a dissolving one and the desorption process is a concentrating one. No conflicts exist with the older pore geometry approach, since ink-well and spherical pores would dissolve more of the solid pore and exhibit greater hysteresis.

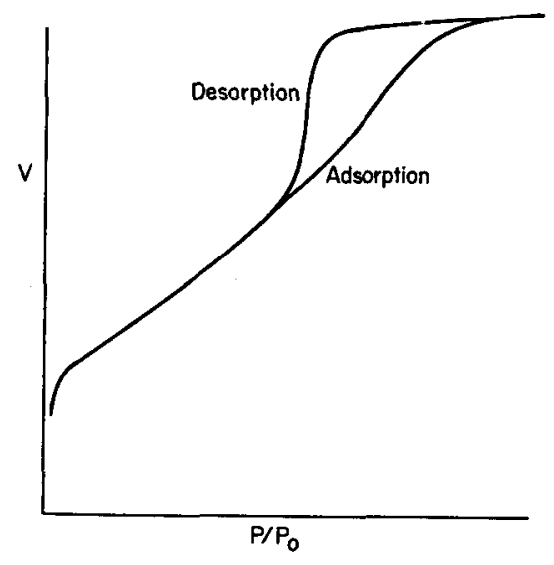

Fig. 3. Adsorption-desorption hysteresis.

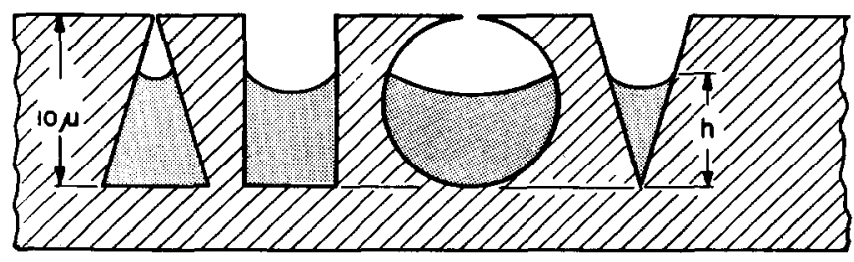

Fig. 4. Subsurface pores of different geometries. 


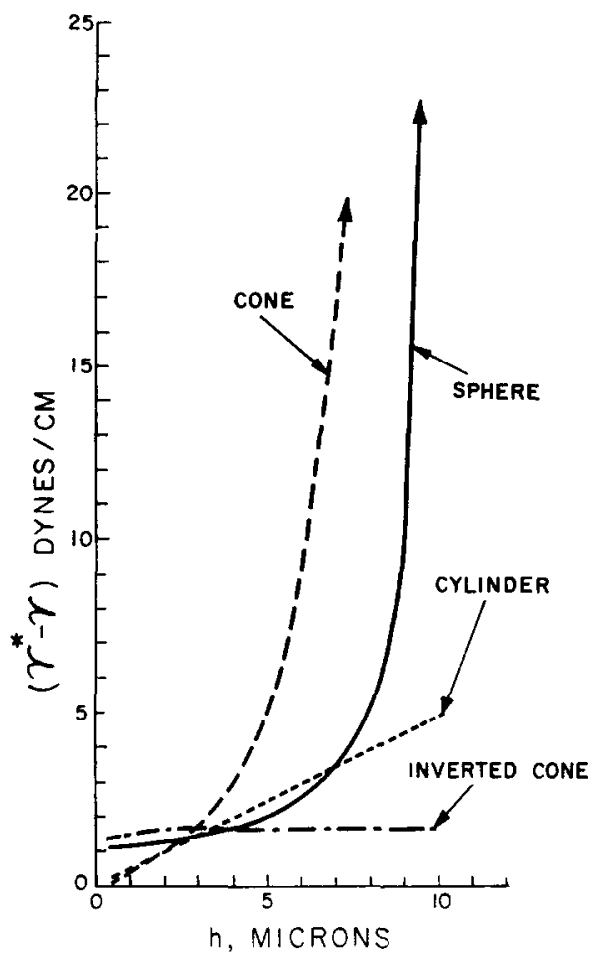

Fig. 5. Changes in surface tension of a liquid of unit surface tension as a function of liquid height.

\section{References}

1) J. L. Shereshefsky, J. Am. Chem. Soc. 50 (1928) 2966.

2) M. Folman and J. L. Shereshefsky, J. Phys. Chem. 59 (1955) 607.

3) T. Gillespie and W. J. Settineri, J. Colloid Chem. 24 (1967) 199; Discussions, H. M. Prince and B. Derjaguin, J. Colloid Chem. 26 (1968) 246.

4) B. V. Derjaguin, N. V. Churaev, N. N. Fedyakin, M. V. Talaev and I. G. Ershova, Izv. Akad. Nauk SSSR 10 (1967) 2178.

5) W. J. O'Brien, Surface Sci. 19 (1970) 387.

6) N. L. Cross and R. G. Picknett, in: Proc. Intern. Conf. Mechanism of Corrosion by Fuel Impurities, Marchwood, England, 1963, p. 383.

7) J. T. Davies and E. K. Rideal, Interfacial Phenomena, 2nd ed. (Academic Press, New York, 1963) pp. 426-428.

8) J. L. Shereshefsky, ibid. (1928).

9) J. H. Hildebrand and R. L. Scott, The Solubility of Nonelectrolytes (Reinhold, New York, 1950) p. 431.

10) S. W. Rabideau and A. E. Florin, Science No. 1969 (1970) 48.

11) R. E. Davies, D. L. Rousseau and R. Board, Chem. Eng. News June 29 (1970) 7.

12) D. L. Rousseau and S. P. Porto, Science 167 (1970) 1715.

13) W. Drost-Hansen, Ind. Eng. Chem. 61 (1969) 10. 